\title{
HOMOGENIZATION OF PLATES WITH PARALLEL CRACKS
}

\author{
Mateusz Holek, Piotr Fedeliński \\ Department of Computational Mechanics and Engineering, Silesian University of Technology \\ Gliwice, Poland \\ mateusz.holek@polsl.pl,piotr.fedelinski@polsl.pl
}

Received: 20 November 2019; Accepted: 26 January 2020

\begin{abstract}
The paper presents an analysis of effective elastic properties of plates with parallel cracks using the finite element method (FEM) and the boundary element method (BEM). Rectangular plates with parallel or inclined cracks to the edges of plates were considered. Different distances between cracks and different angles of cracks were studied. The displacement and traction boundary conditions were applied and their influence on the accuracy of overall properties of cracked material was analysed. The results obtained by the FEM and the BEM were compared.
\end{abstract}

MSC 2010: 74A45, 65N30, 65N38, 74Q20

Keywords: fracture mechanics, crack, finite element method, boundary element method, effective properties, representative volume element

\section{Introduction}

Cracks in materials are usually undesirable and cause a significant reduction in the durability of structures. Mechanics of fracture is becoming an increasingly important field of knowledge, because research related to the discontinuity of materials, which are cracks, allow for a significant extension of product life. Research in this field is mainly focused on cyclic loading systems, when cracks grow very fast. A plate that has cracks can be classified as a non-homogeneous material, because cracks, and especially those that occur in one direction, cause a significant deterioration of the mechanical properties of such structures. One of the micromechanical methods is homogenization, which allows for the replacing properties of a heterogeneous material by effective properties of a homogeneous material.

The effective elastic properties of cracked materials can be calculated using analytical or numerical methods. Nemat-Nasser and Hori [1] studied analytically an influence of crack density, the coefficient of friction and loading conditions on the effective bulk and shear modulus. The self-consistent method was used to take into account the interaction of cracks. Borovik [2] determined the effective elastic properties of a sintered material with pore channels treated as Y-shaped cracks, which created a periodic regular hexagonal network. A unit cell containing 
halves of two adjacent cracks was modeled using the finite element method (FEM). The influence of different dimensions of crack branches on the effective Young modulus and the Poisson ratio was investigated. Sevostianov et al. [3] studied the influence of the microstructure of the sintered metal fibers on elastic properties. The analytical methods: the non-interaction approximation, the effective media approach, the differential approach and the effective field methods were used to derive explicit formulas for calculation of effective properties. The effect of the relative volume of pores, pore shapes and relative length of crack branches, which depend on the temperature of sintering, was analysed. Liu and Graham-Brady [4] derived expressions for the compliance of periodically distributed wingcracks under uniaxial compressive load. An influence of the number of cracks, their size and orientation and the coefficient of friction was studied. The analytical results were compared with the FEM solutions. Dong and Lee [5] used the boundary integral equation method to analyse a doubly periodic array of cracks in an infinite isotropic plate subjected to remote tensile or shear loadings. The boundary integral equations depended on opening displacements of cracks. The effective elastic properties of the equivalent orthotropic material were calculated using a rectangular cell with a single crack. The influence of lengths of the cracks and dimensions of the cells was studied. Linkov and Koshelev [6] presented complex variable singular and hypersingular boundary integral equations for a doubly periodic system of grains, inclusions, holes and cracks. The method was used to analyse stress intensity factors and effective compliances of plates with straight, kinked and semi-circular cracks. Linkov [7] showed the application of the method for computation of stress intensity factors, trajectories of growing wings and compliances of doubly periodic inclined cracks. Various crack distributions were considered in the works.

Fedelinski [8] analysed effective elastic properties for representative volume elements (RVE) with randomly distributed cracks by the boundary element method (BEM). The cracks having the same length, randomly distributed, parallel or randomly oriented were considered. The influence of density of cracks on the effective Young modulus and the effective Poisson ratio was presented. Fedelinski [9] considered sintered metal fibers with voids and branched cracks grooving from their centers. The RVEs containing a large number of regularly distributed branched cracks were investigated. The influence of void shapes and dimensions of the crack branches on elastic properties were investigated. Fedelinski [10] analysed plates with cracks subjected to compressive loadings. The contact forces between crack surfaces were determined using the iterative procedure. An influence of orientation of cracks on overall elastic properties was studied.

The original contribution of this work is an analysis of periodic RVEs with parallel cracks intersecting the boundary subjected to traction or displacement boundary conditions and RVEs with inclined cracks subjected to compression. The effective elastic properties computed for different boundary conditions using the FEM and the BEM are compared.

The plates with cracks are analysed by the finite element method and the boundary element method. The software Ansys 18.2 is used in the finite element analysis. 
The dual boundary element method [11] and the computer code developed by Portela and Aliabadi [12] is applied to solve numerical examples. In order to take into account the contact of crack edges, the computer code was extended [10]. In the FEM analysis, eight-node quadrilateral and in the BEM three-node line elements are applied.

\section{Plates with cracks parallel to the boundary}

Rectangular plates with uniformly distributed straight cracks parallel to the edges of the plate are considered. The periodic representative volume element (RVE) containing 17 internal cracks and 10 edge cracks is shown in Figure 1. The global coordinate system $x_{1}-x_{2}$ is located in the centre of the plate. The dimensions of the RVE are $2 w$ and $2 h$, in the $x_{1}$ and $x_{2}$ direction, respectively. The lengths of the cracks are $2 a$, the distance between the cracks in the $x_{2}$ direction is $b$ and the distance of the centres of the cracks in $x_{1}$ direction is $c$.

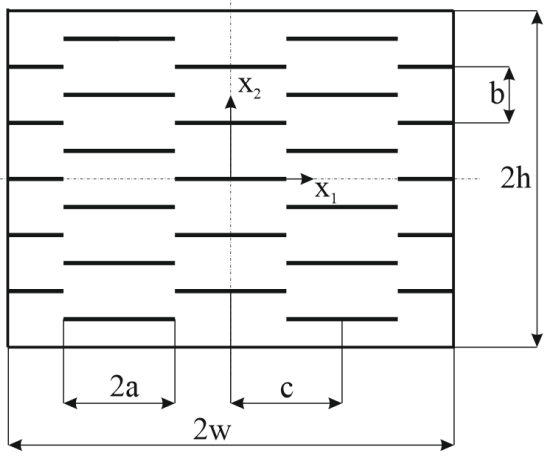

Fig. 1. Dimensions of the plate with cracks parallel to the boundary

Five plates with a different distribution of cracks are studied. The relative distances between the cracks are given in Table 1 . The dimensions of plates are related to the distances between cracks $w=2 c$ and $h=3 b$. The material of plates is linear-elastic, isotropic and homogeneous. For all plates, the Poisson ratio of the material is $v_{0}=0.3$ and the plates are in plane stress conditions.

In the FEM analysis, 400 finite elements and in the BEM analysis 10 boundary elements are used along each crack edge.

The displacements along the external boundaries of the RVE are used to calculate average strains $\bar{\varepsilon}_{i j}$ :

$$
\bar{\varepsilon}_{i j}=\frac{1}{2 A} \int_{\Gamma}\left(u_{i} n_{j}+u_{j} n_{i}\right) d \Gamma,
$$

where $A$ is the area of the RVE, $\Gamma$ is its boundary, $u_{i}$ is the component of the displacement, $n_{i}$ is the component of the unit normal outward vector to the boundary, and $i, j=1,2$ for two-dimensional problems. 
Table 1. Relative distances between cracks

\begin{tabular}{|c|c|c|}
\hline Plate's number & $c / a$ & $b / a$ \\
\hline 1 & 2.0 & 1.0 \\
\hline 2 & 1.5 & 1.0 \\
\hline 3 & 2.5 & 1.0 \\
\hline 4 & 2.0 & 0.5 \\
\hline 5 & 2.0 & 1.5 \\
\hline
\end{tabular}

The tractions along the external boundaries of the RVE are used to calculate average stresses $\bar{\sigma}_{i j}$ :

$$
\bar{\sigma}_{i j}=\frac{1}{A} \int_{\Gamma} t_{i} x_{j} d \Gamma,
$$

where $t_{i}$ is the component of the traction.

\subsection{Traction boundary conditions}

The plate is simply supported along the vertical axis of symmetry allowing free extension, as shown in Figure 2. The plate is loaded by uniformly distributed vertical tractions $p_{2}$ applied along the horizontal edges.

The normalised displacements of the upper $u_{2} / u_{20}$ and right edge $u_{1} / u_{10}$ of the plate 1, computed by the FEM and the BEM, are shown in Figure 3. The displacements are normalised with respect to the displacements of the edges of the plate without cracks $u_{10}$ and $u_{20}$. The normalised displacements are shown as functions of normalised coordinates $x_{1} / w$ and $x_{2} / h$.

The displacements of the upper edge computed by the BEM are larger than the FEM displacements, as shown in Figure 3a. The largest vertical displacements are for the boundary near the cracks. Very good agreement of horizontal displacements computed by the FEM and the BEM for the right edge can be seen in Figure $3 \mathrm{~b}$. The largest horizontal displacements are for the corners of the plate.

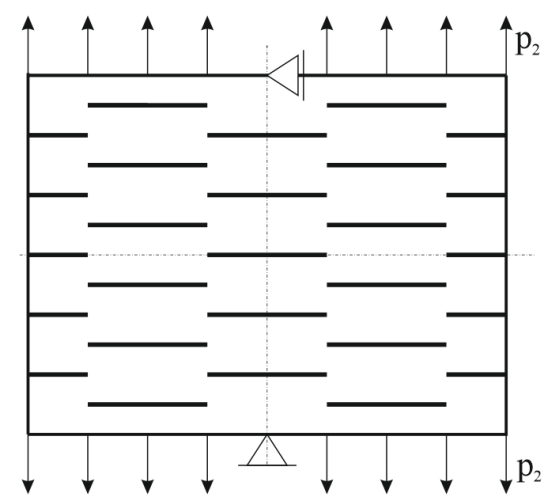

Fig. 2. Traction boundary conditions for the plate with parallel cracks 

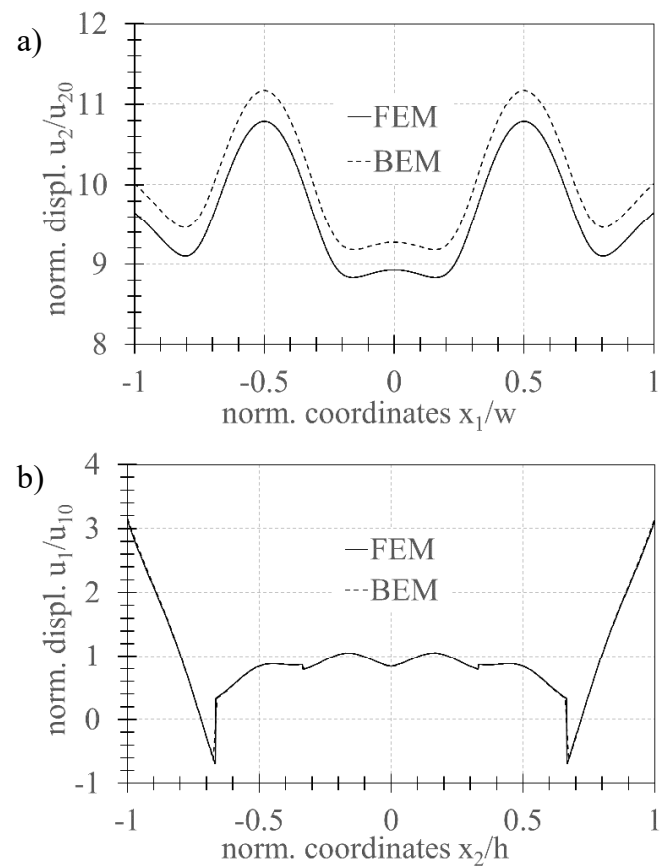

Fig. 3. Normalised displacements of edges of the plate 1: a) upper edge, b) right edge

The average strains are computed using Eq. (1). They are used to calculate the effective Young modulus $E_{2}$ and the Poisson ration $v_{12}$. The normalised effective Young modulus $E_{2} / E_{0}$ and the effective Poisson coefficient $v_{12} / v_{0}$ of plates are shown in Table 2, where $E_{0}$ and $v_{0}$ are the Young modulus and the Poisson ratio of the continuous material in plane stress conditions, respectively. The relative differences of the properties obtained by two methods with respect to the properties of the continuous material $\left[E_{2}(\mathrm{FEM})-E_{2}(\mathrm{BEM})\right] / E_{0}$ and $\left[v_{12}(\mathrm{FEM})-v_{12}(\mathrm{BEM})\right] / v_{0}$ are also shown in Table 2.

Table 2. Effective properties of the plates for the traction boundary conditions

\begin{tabular}{|c|c|c|c|c|}
\hline Plate's number & Properties & FEM & BEM & Difference [\%] \\
\hline \multirow{3}{*}{1} & $E_{2} / E_{0}$ & 0.0969 & 0.0935 & 0.34 \\
\cline { 2 - 5 } & $v_{12} / v_{0}$ & 0.0967 & 0.0892 & 0.75 \\
\hline \multirow{2}{*}{2} & $E_{2} / E_{0}$ & 0.0289 & 0.0281 & 0.08 \\
\cline { 2 - 5 } & $v_{12} / v_{0}$ & 0.0285 & 0.0182 & 1.03 \\
\hline \multirow{3}{*}{3} & $E_{2} / E_{0}$ & 0.2194 & 0.2152 & 0.42 \\
\cline { 2 - 5 } & $v_{12} / v_{0}$ & 0.2746 & 0.2611 & 1.35 \\
\hline \multirow{3}{*}{4} & $E_{2} / E_{0}$ & 0.0666 & 0.0629 & 0.37 \\
\cline { 2 - 5 } & $v_{12} / v_{0}$ & 0.0659 & 0.0573 & 0.86 \\
\hline \multirow{2}{*}{5} & $E_{2} / E_{0}$ & 0.1031 & 0.1006 & 0.25 \\
\cline { 2 - 5 } & $v_{12} / v_{0}$ & 0.1028 & 0.0914 & 1.14 \\
\hline
\end{tabular}


One can notice a significant influence of crack spacing on values of effective Young moduli or Poisson ratios. The most stiffest plate is plate 3 for which $c / a$ value is the largest. The separation of cracks in the $x_{1}$ direction results in the forming of areas of material without discontinuities. They significantly increase the stiffness of the material. On the other hand, the plate with the smallest values of the effective Young's modulus and effective Poisson's ratio is the plate 2, in which the cracks overlap each other in the $x_{1}$ direction. This causes significant displacement values on the outer edges of the plate, which decreased the Young modulus. The relative Poisson ratios are smaller than the relative Young moduli except for the stiffest plate 3 .

The effective elastic properties, obtained by the FEM are larger than the properties computed by the BEM for each plate. Good agreement of results can be seen for the Young moduli, where the relative difference is $0.08-0.42 \%$. The Poisson ratio shows less consistent results for the FEM and the BEM. The relative difference is $0.75-1.35 \%$. The accuracy of the Young modulus is better than the Poisson ratio because displacements of two edges are used to compute the Young modulus and displacements of all edges to compute the Poisson ratio. The discrepancies in the results are also affected by the differences in the structure discretization.

\subsection{Displacement boundary conditions}

The same RVEs are analysed using the prescribed displacement boundary conditions on all edges of the plates. Obtaining effective material properties is a much more complex process than for plates with traction boundary conditions.

In order to obtain effective properties, two types of boundary conditions shown in Figure 4 are applied. Two edges of the plates are supported and can move only in the directions of the edges, and on the remaining edges uniform displacements are given.

a)

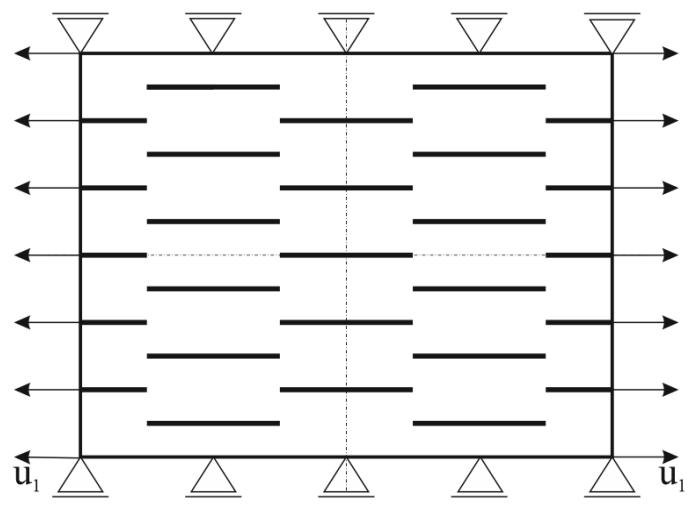

b)

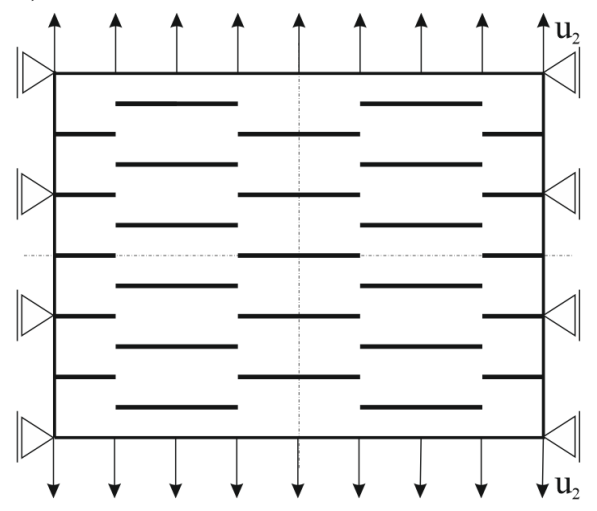

Fig. 4. Displacement boundary conditions for the plate with parallel cracks: a) applied displacements $u_{1}$, b) applied displacements $u_{2}$ 
In this method, the averaged stresses are computed using Eq. (2). For the applied displacements $u_{1}$ in the $x_{1}$ direction (Fig. 4a), the relations between average strains and stresses is expressed by the following equations:

$$
\begin{aligned}
& \bar{\varepsilon}_{1}^{a}=\frac{1}{E_{1}} \bar{\sigma}_{1}^{a}-\frac{v_{12}}{E_{2}} \bar{\sigma}_{2}^{a}, \\
& 0=-\frac{v_{21}}{E_{1}} \bar{\sigma}_{1}^{a}+\frac{1}{E_{2}} \bar{\sigma}_{2}^{a} .
\end{aligned}
$$

For the applied displacements $u_{2}$ in the $x_{2}$ direction (Fig. 4b) the relations between average strains and stresses are expressed by the following equations:

$$
\begin{gathered}
0=\frac{1}{E_{1}} \bar{\sigma}_{1}^{b}-\frac{v_{12}}{E_{2}} \bar{\sigma}_{2}^{b}, \\
\bar{\varepsilon}_{2}^{b}=-\frac{v_{21}}{E_{1}} \bar{\sigma}_{1}^{b}+\frac{1}{E_{2}} \bar{\sigma}_{2}^{b},
\end{gathered}
$$

where the superscript $a$ denotes stresses for the first type and the superscript $b$ stresses for the second type of boundary conditions.

The equations (3)-(6) are used to calculate effective properties of the material:

$$
\begin{gathered}
E_{1}=\frac{\bar{\sigma}_{2}^{b} \bar{\sigma}_{1}^{a}-\bar{\sigma}_{2}^{a} \bar{\sigma}_{1}^{b}}{\bar{\varepsilon}_{1}^{a} \bar{\sigma}_{2}^{b}}, \\
E_{2}=\frac{\bar{\sigma}_{2}^{b} \bar{\sigma}_{1}^{a}-\bar{\sigma}_{2}^{a} \bar{\sigma}_{1}^{b}}{\bar{\varepsilon}_{2}^{b} \bar{\sigma}_{1}^{a}}, \\
v_{12}=E_{2} \frac{\bar{\varepsilon}_{1}^{a} \bar{\sigma}_{1}^{b}}{\bar{\sigma}_{2}^{b} \bar{\sigma}_{1}^{a}-\bar{\sigma}_{2}^{a} \bar{\sigma}_{1}^{b}}, \\
v_{21}=E_{1} \frac{\bar{\varepsilon}_{2}^{b} \bar{\sigma}_{2}^{a}}{\bar{\sigma}_{2}^{b} \bar{\sigma}_{1}^{a}-\bar{\sigma}_{2}^{a} \bar{\sigma}_{1}^{b}} .
\end{gathered}
$$

The normalised tractions along the upper $p_{2} / p_{20}$ and the right edge $p_{1} / p_{10}$ of the plate 1 computed by the FEM and the BEM are shown in Figure 5. The tractions are normalised with respect to the tractions for the plate without cracks $p_{10}$ and $p_{20}$. The tractions computed by the FEM are slightly larger than the BEM tractions. The smallest tractions are along the boundary near the cracks.

The normalised effective Young modulus $E_{2} / E_{0}$ and the effective Poisson coefficient $v_{12} / v_{0}$ of plates are shown in Table 3 . The relative differences are computed in the same way as for the traction boundary conditions. The Young modulus $E_{1}$ 
and the Poisson ratio $v_{21}$ are not given because they have exactly the same values as for the continuous material, namely $E_{1}=E_{0}$ and $v_{21}=v_{0}$.

Table 3. Effective properties of the plates for the displacement boundary conditions

\begin{tabular}{|c|c|c|c|c|}
\hline Plate's number & Properties & FEM & BEM & Difference [\%] \\
\hline \multirow{3}{*}{1} & $E_{2} / E_{0}$ & 0.1099 & 0.1044 & 0.55 \\
\cline { 2 - 5 } & $v_{12} / v_{0}$ & 0.1065 & 0.0793 & 2.72 \\
\hline \multirow{2}{*}{2} & $E_{2} / E_{0}$ & 0.0322 & 0.0327 & -0.05 \\
\cline { 2 - 5 } & $v_{12} / v_{0}$ & 0.0373 & 0.0267 & 1.06 \\
\hline \multirow{3}{*}{3} & $E_{2} / E_{0}$ & 0.2728 & 0.2808 & -0.80 \\
\cline { 2 - 5 } & $v_{12} / v_{0}$ & 0.2513 & 0.2817 & -3.04 \\
\hline \multirow{3}{*}{4} & $E_{2} / E_{0}$ & 0.0497 & 0.0552 & -0.55 \\
\cline { 2 - 5 } & $v_{12} / v_{0}$ & 0.0494 & 0.0556 & -0.62 \\
\hline \multirow{2}{*}{5} & $E_{2} / E_{0}$ & 0.1641 & 0.1673 & -0.32 \\
\cline { 2 - 5 } & $v_{12} / v_{0}$ & 0.0750 & 0.1611 & -8.61 \\
\hline
\end{tabular}

Good agreement of results can be seen for the Young moduli, where the absolute value of the relative difference is $0.05-0.80 \%$. The Poisson ratio shows less consistent results for the FEM and the BEM. The relative difference is $0.62-8.61 \%$. The Young modulus computed using the displacement boundary conditions is larger than the modulus computed using the traction boundary conditions except for the most flexible plate 4 .
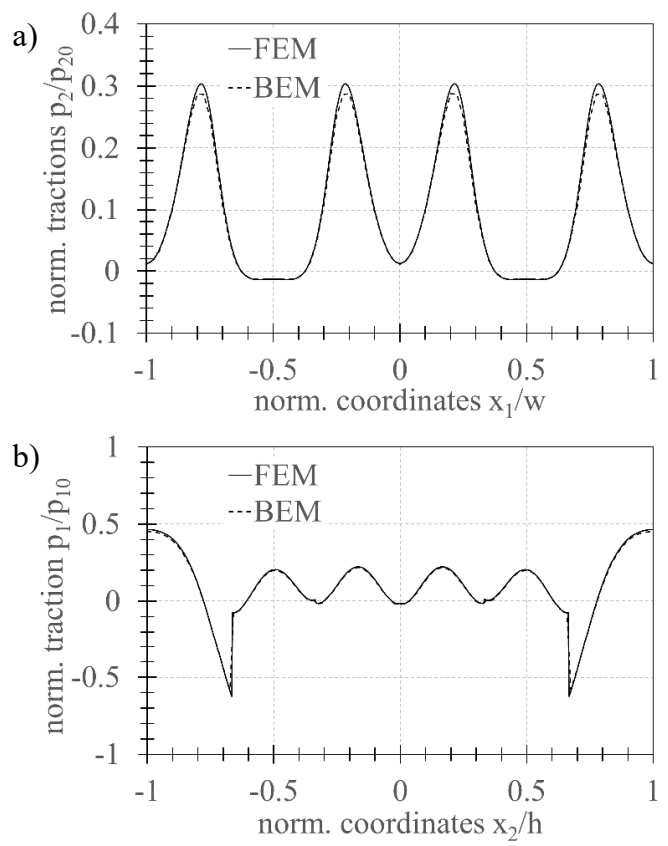

Fig. 5. Normalised tractions along edges of the plate 1: a) upper edge, b) right edge 


\section{Plates with parallel cracks inclined to the boundary}

Square plates, shown in Figure 6, having the dimensions $2 w=2 h$ with 21 parallel cracks inclined at the angle $\alpha$ with respect to the axis $x_{1}$ are analysed [10]. The ratio of the crack length to the edge length is $a / w=1 / 8$. The distances between the cracks are $b=c=2 \sqrt{2} a$. Three different orientations of the cracks are considered, namely $\alpha=30^{\circ}, 45^{\circ}$ or $60^{\circ}$. The plates are in plane strain conditions and the Poisson ratio of the material is $v=0.3$. The plates are subjected to the horizontal compressive tractions $p_{1}$. Along each edge of the crack, 10 boundary elements or 80 finite elements are used.

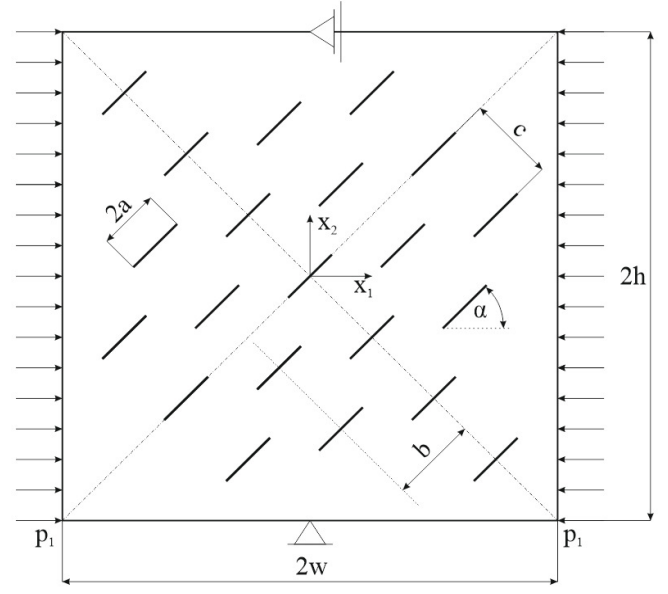

Fig. 6. Plate with inclined parallel cracks

The plates under compression are analysed by neglecting or by taking into account the contact of crack edges. Contact features in the Ansys software are determined as frictionless, surface to surface with the normal Lagrange formula. These settings meant that penetration and opening are close to zero. The normalised effective Young modulus $E_{1} / E_{0}$ and the effective Poisson coefficient $v_{21} / v_{0}$ of plates are shown in Table 4, where $E_{0}$ and $v_{0}$ are the Young modulus and the Poisson ratio of the continuous material in plane strain conditions, respectively. The relative differences are computed as for the traction boundary conditions in the first numerical example. Good agreement of the results can be seen for the Young moduli, where the absolute value of relative difference is $0.2-1.3 \%$. The Poisson ratio shows less consistent results for the FEM and the BEM. The absolute value of relative difference is $0.3-1.8 \%$.

It can also be seen that for the angles $30^{\circ}$ and $60^{\circ}$ the effective Young moduli and the Poisson ratios, computed with contact, show very high similarity.

In this example cracks do not intersect the boundaries of the RVEs and variations of displacements along the edges are much smaller than in the first example. Therefore the accuracy of displacements, average strains and effective properties is better. 
Table 4. Effective properties of the plates with inclined parallel cracks

\begin{tabular}{|c|c|c|c|c|c|c|c|}
\hline \multicolumn{2}{|c|}{ Angle $\alpha$} & \multicolumn{2}{c|}{$30^{\circ}$} & \multicolumn{2}{c|}{$45^{\circ}$} & \multicolumn{2}{c|}{$60^{\circ}$} \\
\hline \multicolumn{2}{|c|}{ Contact } & no & yes & no & yes & no & yes \\
\hline \multirow{2}{*}{$E_{1} / E_{0}$} & FEM & 0.859 & 0.895 & 0.767 & 0.877 & 0.666 & 0.895 \\
\cline { 2 - 8 } & BEM & 0.866 & 0.900 & 0.763 & 0.875 & 0.679 & 0.899 \\
\hline \multicolumn{2}{|c|}{ Difference $[\%]$} & -0.7 & -0.5 & 0.4 & 0.2 & -1.3 & -0.4 \\
\hline \multirow{2}{*}{$v_{21} / v_{0}$} & FEM & 0.828 & 1.140 & 0.723 & 1.163 & 0.641 & 1.140 \\
\cline { 2 - 8 } & BEM & 0.840 & 1.130 & 0.719 & 1.166 & 0.659 & 1.131 \\
\hline \multicolumn{2}{|c|}{ Difference $[\%]$} & -1.2 & 1.0 & 0.4 & -0.3 & -1.8 & 0.9 \\
\hline
\end{tabular}

\section{Conclusions}

Representative volume elements with parallel or inclined cracks to the edges of the element were considered in the paper. The influence of distance between cracks, their orientation and the influence of crack edge contact on effective Young's moduli and Poisson's ratios were investigated. Stress or displacement boundary conditions were given to determine effective properties. The materials were analysed by the finite and boundary element methods. Better consistency of results for Young's moduli than for Poisson's coefficients was obtained. It is particularly difficult to obtain accurate results for periodic representative volume elements in which cracks cut the edges of the element due to large changes in displacements and tractions on the edges of the element.

The largest Young's moduli were obtained for plates in which there were areas of continuous load-bearing material between cracks. Materials with cracks have greater stiffness in compression than in tension.

\section{Acknowledgement}

The scientific research is financed by National Science Centre, Poland, in years 2016-2020, grant no. 2015/19/B/ST8/02629.

\section{References}

[1] Nemat-Nasser, S., \& Hori, M. (1999). Micromechanics: Overall Properties of Heterogeneous Materials. Elsevier.

[2] Borovik, V.G. (2010). Modelling the effective elastic properties of materials with parallel pore channels with Y-shaped cross-sections. Powder Metallurgy and Metal Ceramics, 49, 272-279.

[3] Sevostianov, I., Picazo, M., \& Garcia, J.R. (2011). Effect of branched cracks on the elastic compliance of a material. International Journal of Engineering Science, 49, 1062-1077. 
[4] Liu, J., \& Graham-Brady, L. (2016). Effective anisotropic compliance relationships for wingcracked brittle materials under compression. International Journal of Solids and Structures, 100-101, 151-168.

[5] Dong, C.Y., \& Lee, K.Y. (2005). Numerical analysis of doubly periodic array of cracks/rigidline inclusions in an infinite isotropic medium using the boundary integral equation method. International Journal of Fracture, 133, 389-405.

[6] Linkov, A.M., \& Koshelev, V.F. (1999). Complex variables BIE and BEM for a plane doubly periodic system of flaws. Journal of the Chinese Institute of Engineers, 22, 709-720.

[7] Linkov, A.M. (2002). Boundary Integral Equations in Elasticity Theory. Kluwer Academic Publishers.

[8] Fedelinski, P. (2011). Analysis of representative volume elements with random microcracks. Chapter 17, in Computational Modelling and Advanced Simulation, Computational Methods in Applied Sciences 24, ed. J. Murin et al. Springer Science+Business Media B.V., 333-341.

[9] Fedelinski, P. (2017). Effective properties of sintered materials with branched cracks. American Institute of Physics Conference Proceedings 1922, 030008-1-8.

[10] Fedelinski, P. (2019). Boundary element analysis of cracks under compression. American Institute of Physics Conference Proceedings 2078, 020009-1-7.

[11] Portela, A., Aliabadi, M.H., \& Rooke, D.P. (1992). The dual boundary element method: effective implementation for crack problems. International Journal for Numerical Methods in Engineering, 33, 1269-1287.

[12] Portela, A., \& Aliabadi, M.H. (1993). Crack Growth Analysis using Boundary Elements. Computational Mechanics Publications. 